\title{
Analysis of the scope of cooperative bandwidth sharing among mobile routers in vehicular networks
}

\author{
Lai Tu', Shih-Yang Lin² and Chung-Ming Huang ${ }^{2 *}$
}

\begin{abstract}
This paper investigates the optimal cooperation scope (OCS) for cooperative bandwidth sharing (CBS) in vehicular networks. An analytical model, which comprises the network model, relay schemes, cost model, and vehicular flow model, is presented. Three relay schemes based on optimal single path relay and opportunistic relay for multi-hop CBS are proposed and studied. The OCS is solved as the maximum physical distance or hops between the bandwidth requester and bandwidth helper in the optimal solution to maximize the CBS benefit. OCSs for different environments and with different relay schemes are given in the paper. The results indicate that the OCS is sensitive to the cost weight and may also be larger when using a proper opportunistic relay-based scheme than when using the optimal single path-based one. The results also show that the vehicular density does not affect the optimal cooperation scope much but can result in different CBS benefit. The model and findings of the relations between OCS and the running environment revealed in this paper can be used as a reference for parameter choosing when designing the CBS system in vehicular networks.
\end{abstract}

Keywords: Vehicular networks; Cooperative bandwidth sharing; Relay scheme; Cooperation scope; Vehicular flow model

\section{Introduction}

Recent advances in vehicular technologies have promoted new wireless technologies for vehicles' internetworking [1]. In particular, the dedicated short-range communications (DSRC) technology, which is a variant of IEEE 802.11a, is designed to operate within a frequency band $(5.9 \mathrm{GHz})$ licensed solely for the purpose of vehicular communications and is being optimized for operation within the high-speed vehicular environment [2]. The DSRC technology has been studied as an inter-vehicle communications platform for applications like collision avoidance [3], automated highway systems [4], and passenger teleconferencing. Meanwhile, Internet access on vehicles is usually provided by some device called Mobile Router, which generally has two wireless interfaces for different purposes $[5,6]$. The wireless wide area network (WWAN) interface usually uses $3 \mathrm{G}$ or LTE, WiFi, or

*Correspondence: huangcm@locust.csie.ncku.edu.tw

2 Department of CSIE, National Cheng Kung University, Tainan 701, Taiwan

Full list of author information is available at the end of the article modified WiFi technology to connect to the corresponding infrastructure, and the wireless local area network (WLAN) interface mostly adopts WiFi to supply local network connection on vehicle terminals.

Therefore, it is quite possible that in a future vehicle, an onboard unit (OBU) integrates all of these techniques for different purposes and works cooperatively to maximize the efficiency. In this paper, we investigate one of such issues, i.e., the optimal cooperative bandwidth sharing for mobile routers in vehicular networks. More specifically, we focus on the cooperation among nodes using WWAN and $\mathrm{V} 2 \mathrm{~V}$ wireless interfaces.

Cooperative bandwidth sharing (CBS) is motivated by three facts that exist in wireless mobile networks:

1. The WiFi-based technologies suffer from connectivity disruptions in the vehicular environment $[7,8]$, while data rate of $3 \mathrm{G}$ or LTE degrades quite much when vehicles are moving fast or users in the cell are crowded. Thus, none of the

\section{至 Springer}

(c) 2014 Tu et al: licensee Springer. This is an Open Access article distributed under the terms of the Creative Commons

Attribution License (http://creativecommons.org/licenses/by/2.0), which permits unrestricted use, distribution, and reproduction in any medium, provided the original work is properly credited. 
current wireless technologies can provide perfect performance for WWAN in the vehicular environment.

2. Internet traffic load and pattern can be rather different from node to node. For example, Internet TV service may be provided on a bus, and some users in another car may like surfing the Internet while there happens to be no Internet traffic from a third truck.

3. Although DSRC supports inter-vehicle communication, most of its applications are limited to a specific safety or infotainment purpose; otherwise, the DSRC device is usually idle. Few studies consider DSRC as an auxiliary means to enhance general Internet access despite its communication capability, which is really some kind of waste.

As a result, we consider an approach to enable bandwidth sharing among vehicles via DSRC communications. The onboard mobile router that is short of bandwidth borrows bandwidth from one or more mobile routers in a scope whose traffic is light enough to have extra bandwidth. So the WWAN bandwidth resource has been pooled together and fully utilized by mobile routers.

While cooperation among colocated mobiles is not a new concept in mobile networks (see Section 2 for literature review), it is rarely studied in the vehicular environment. Due to the difference in the vehicular environment, point-to-point link between two vehicles is not stable. Consequently, there may not be many collaborators in one hop range. However, if multi-hop cooperation is enabled, it may be a problem whether the relay cost will overwhelm the benefit from cooperation or not. So, in this paper, we are trying to answer the question: Is it worthy to use multihop bandwidth sharing for mobile routers in the vehicular environment? If the answer is positive, a further question is to be answered: How many hops will be the upper bound?

To answer the aforementioned questions, we build an analytical model that considers both networking model and vehicular flow model. We formulate the CBS problem into an optimization problem and analyze the cooperation scope (CS) in the optimal solution. A distributed algorithm to solve the CBS problem is also given as a reference for practical implementation. The results show that the optimal cooperation scope is sensitive to the cost weight and may also vary with relay schemes. The optimal cooperation scopes in different scenarios are given which can guide parameter setting in designing a CBS system. To the best of our knowledge, the work in this paper is the first attempt to model cooperative bandwidth sharing in the vehicular environment and it also gives some new findings in the CBS problem.
The rest of the paper is organized as follows: After a general review of related work in Section 2, the model details are elaborated in Section 3. Section 4 briefly introduces a distributed algorithm for practical implementation. Numeric results are given and analyzed in Section 5, followed by the conclusion in the last section.

\section{Related work}

Similar researches on aggregating bandwidth of multiple interfaces or mobiles' cooperation to improve efficiency have been investigated in prior work [9-21].

To support Internet connectivity in vehicular environments, both academics and standard organizations have made their efforts on the state-of-the-art network mobility (NEMO) architecture [9-11]. The NEMO Basic Support (NEMO-BS) [10] and later proposed improved solutions $[12,22]$ provided possibility of seamless communications in vehicular environments. The Mobile IP (MIP)-based solutions and their extensions are usually preferred in NEMO due to their scalability and wide adoption [11,23,24]. For instance, the Proxy Mobile IPv6 (PMIPv6)-based NEMO (P-NEMO) and fast P-NEMO can achieve low traffic cost and handover latency for vehicular networks $[24,25]$. To provide uninterrupted Internet connection, application of a multi-homing technique in NEMO is studied in $[14,26]$.

BAG [15] motivated the advantages of simultaneous use of multiple interfaces and proposes a network-layer architecture that enables such use. MAR [16] makes use of the multiplicity of the wireless networks available by dynamically instantiating new channels based on traffic demand, aggregating the bandwidth and dynamically shifting load from poor quality to better quality channels.

Kandula et al. [17] focused on load balancing for more flexible and efficient allocation of resources, thereby extending the lifetime of a network. Sharma et al. [18] proposed a multi-path transport protocol, based on a carefully crafted set of enhancements to TCP, that effectively utilizes the available bandwidth and diversity provided by heterogeneous, lossy wireless paths.

FatVAP [19] introduced an 802.11 driver that aggregates the bandwidth available at accessible access points (APs) and also balances their load. FatVAP challenges the fact that APs often provide high-speed wireless connectivity but access the Internet via independent, relatively lowspeed DSL or cable modem links. If we regard a mobile node with a V2V interface as an AP and an Internet application as a node in the WLAN, the scenarios considered in FatVAP are similar to ours where in both exists bandwidth unbalance.

Inspired by bridging the gap between the range-speed dichotomy of WWAN and WLAN, Ganesh et al. [20] presented COMBINE, a system for collaborative downloading. COMBINE enables mobile devices that are within 
the WLAN range to pool together their WWAN links and thus significantly increases the effective speed available to them. CACBR [27] presented a context-aware community based routing for intermittently connected networks. It exploited the social-based movement pattern and the context-awareness of nodes for efficient message delivery in delay-tolerant networks.

In [28], the authors exploited the potential of smart phones in proximity cooperatively, using their resources to reduce the demand on the cellular infrastructure, through a decision framework called RACE (Resource Aware Collaborative Execution). RACE enables the use of other mobile devices in the proximity as mobile data relays.

In applications of bandwidth aggregation or bandwidth sharing, video streaming is the most common one that benefits multi-sourcing. In [29], the authors provided a unique solution by proposing a new multisource streaming strategy specifically tailored for nextgeneration mobile networks for delivering multimedia services to mobile users.

These works consider different aspects in cooperation coordination for collaborative communication and are mostly like bandwidth borrow schemes, and most of them focus on low mobility scenarios or one-hop scenarios which are quite different in the vehicular environment.

Some other work discussed P2P cooperation in networking on public transport. Liam et al. [30] proposed a new content source selection scheme for single-hop, peer-to-peer based content sharing on public transport. The scheme aims to identify, among colocated peers that have relevant content, the one that has the highest chance to remain colocated long enough for data transfers to complete. However, the scenario also focused on colocated mobiles (meeting by chance) in the same vehicle.

C5 [31] introduced a collaborative content fetching scheme for groups of mobile subscribers with common characteristics. C5 employed a small-scale P2SP framework of a hybrid mobile network which considers possible concurrent mobile Internet traffic to maximize the utility of WWAN links and supports MAC layer multicast in community.

To sum up, these studies discussed the following: (i) solutions and analysis of supporting network mobility in vehicular environment [9-14], (ii) resource aggregation and allocation of multiple interfaces in mobile networks $[15-17,19]$, or (iii) group formation and coordination $[20,21,28]$, or (iv) P2P content sharing among mobiles on one vehicle $[30,31]$. Most of them focus on system implementation and scheme design. In this paper, we bring a new issue and consider the analytical model to formulate the problem.

\section{System model}

In this section, we outline the abstract model of bandwidth sharing among mobile routers in vehicular networks. Figure 1 illustrates a typical scenario of CBS, where a number of vehicles with mobile routers provide Internet access to the onboard users and cooperatively share their excessive bandwidth to insufficient neighbors. We focus on the optimal V2V (vehicle-tovehicle) collaborations among vehicles, and in the model analysis, we simplify the routers' accessing to Internet and provision for onboard users as the source and sink of a traffic flow. We elaborated the abstract model as follows.

\subsection{Target scenario and assumptions}

Before we formulate the problem, we first name the elements and list some assumptions for the characteristics of the operating environment:

- We assume that all mobile routers in the CBS scenario have at least three wireless interfaces: (i) WLAN as the gateway for onboard subscribers, (ii) WAVE for V2V communications, and (iii) 3G/LTE connection as the WWAN for Internet access.

- A mobile router that has sufficient WWAN bandwidth (named the helper) can help the nodes that lack in WWAN bandwidth (named the requester) with its excessive bandwidth on their requests.

- The cooperation scope is considered in two different representations. One is the number of hops of the transmission between the source and destination, and the other is the physical distance between the source and destination nodes. The latter is used due to the number of hops of the transmissions between two same nodes, which may vary by chance in some opportunistic routing schemes. The maximum CS in the optimal cooperative bandwidth sharing solution is considered as the optimal cooperation scope, OCS.

- The cooperative bandwidth sharing can be employed between any helper and requester in a range of CS via either direct link or multi-hop communication. The mobile router that is involved in the traffic relay between a helper and a requester is named forwarder. Note that a helper or a requester can be a forwarder for another helper-requester pair as well. We also assume that a requester is able to find an optimal route to the helper when multi-hop relay is applied.

- It does not matter whether mobiles in the CS are customers of the same WWAN ISP or not, but we assume that each WWAN connection uses an orthogonal channel and there is little interference in WWAN connection among mobiles, despite whether they are from the same ISP or not. We further 


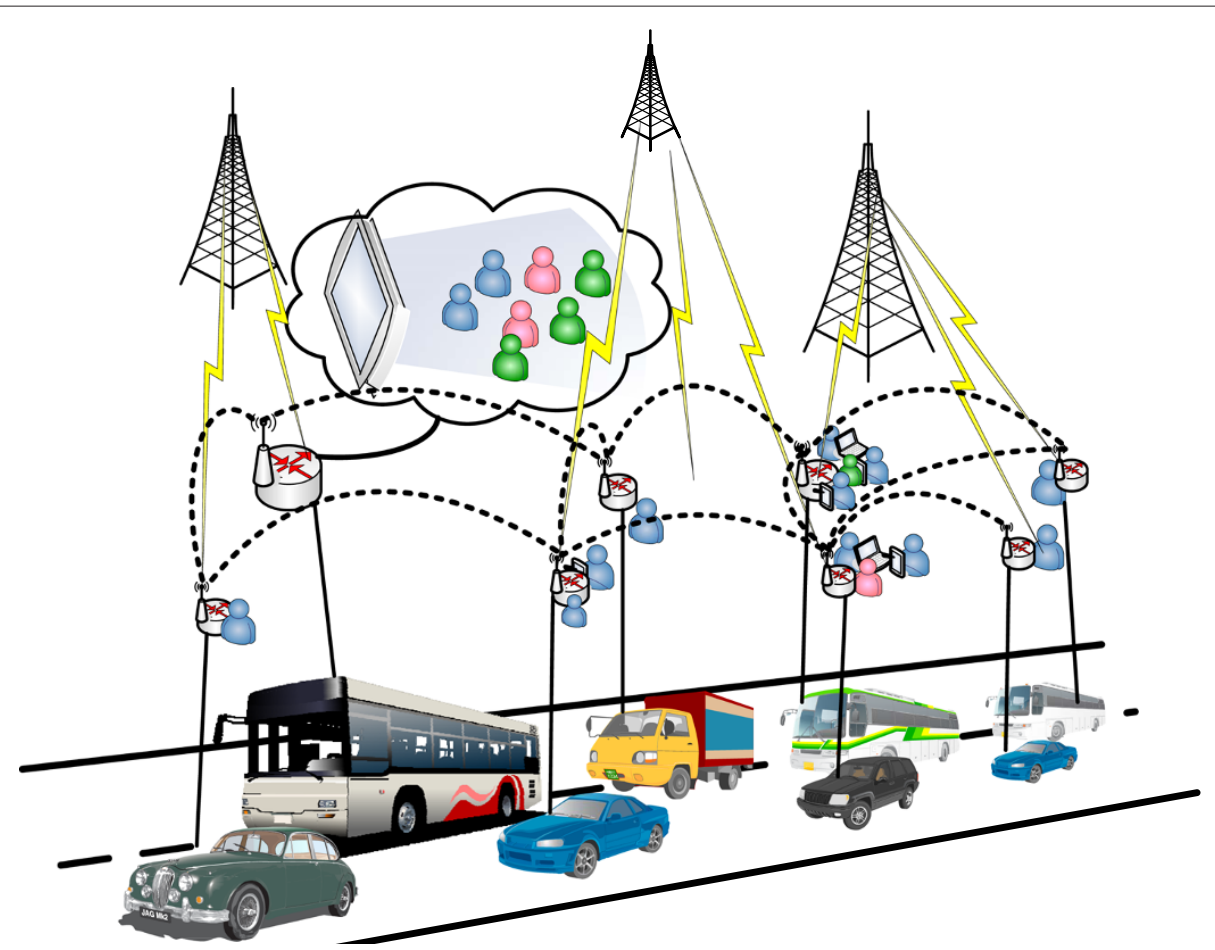

Figure 1 A typical scenario of CBS.

assume that the number of mobiles in a CBS scenario does not exceed the capacity of the WWAN tower.

- Any mobile node is aware of the position and velocity of any other node in its CS, which can be used to help a mobile node to find a route and estimate the cost from the requester to the helper. This is also feasible as current DSRC or WAVE protocol running in the OBU supports multi-channel communication and usually reserves a dedicated broadcast channel to disseminate related information for safety applications, which usually include the vehicle's position and velocity.

This paper focuses on the analytical model of the optimal scope for bandwidth sharing. To make the application feasible for practical engineering, the state-of-the-art network architectures for different interfaces can be used. We simplify the bandwidth sharing operations as traffic forwarding in a resource controllable mobile router. For the Internet connectivity, it is only related with the WWAN interface. We can employ the existing NEMO architecture for the routers connecting the RSU. For the other two interfaces, if the router is a helper, it acts like a gateway to forward the traffic both from WLAN for the users in the vehicle and from $\mathrm{V} 2 \mathrm{~V}$ interface for the neighboring requester. If the router is a requester, it forwards part of the WLAN traffic to WWAN and part of them to $\mathrm{V} 2 \mathrm{~V}$ interface to a helper. Traffic among vehicles in V2V interfaces operates in ad hoc model. A CBS calculation module takes charge of determining the cooperative scope and bandwidth reallocation scheme. The V2V communications evolves the reallocated Internet traffic between helper and requester and the control packages for CBS calculation. The system architecture for such scenario can be illustrated as shown in Figure 2.

Upon the aforementioned assumptions and architecture, we consider the bandwidth sharing problem among a number of mobile nodes in a range of CS. As the bandwidth of every helper may be shared by multiple or different mobiles, the key issue is to find an optimal bandwidth resource allocation solution for different pairs of requesters and helpers. The optimal resource allocation may also be concerned with vehicular flow and networking model. We discuss them in the following subsections. Table 1 lists symbols and terms used in the model.

\subsection{Problem formulation}

There are several requirements for the rate allocation algorithm: (i) It should allocate the bandwidth resources for all mobiles to achieve a maximum utilization of all available WWAN resources. (ii) It should allocate the resource in a fair way. (iii) Communication overhead needs to be minimized for the algorithm.

To do so, we formulate the rate allocation problem into a distributed multi-objective optimization problem as follows. We defined two main objectives of the algorithm as 


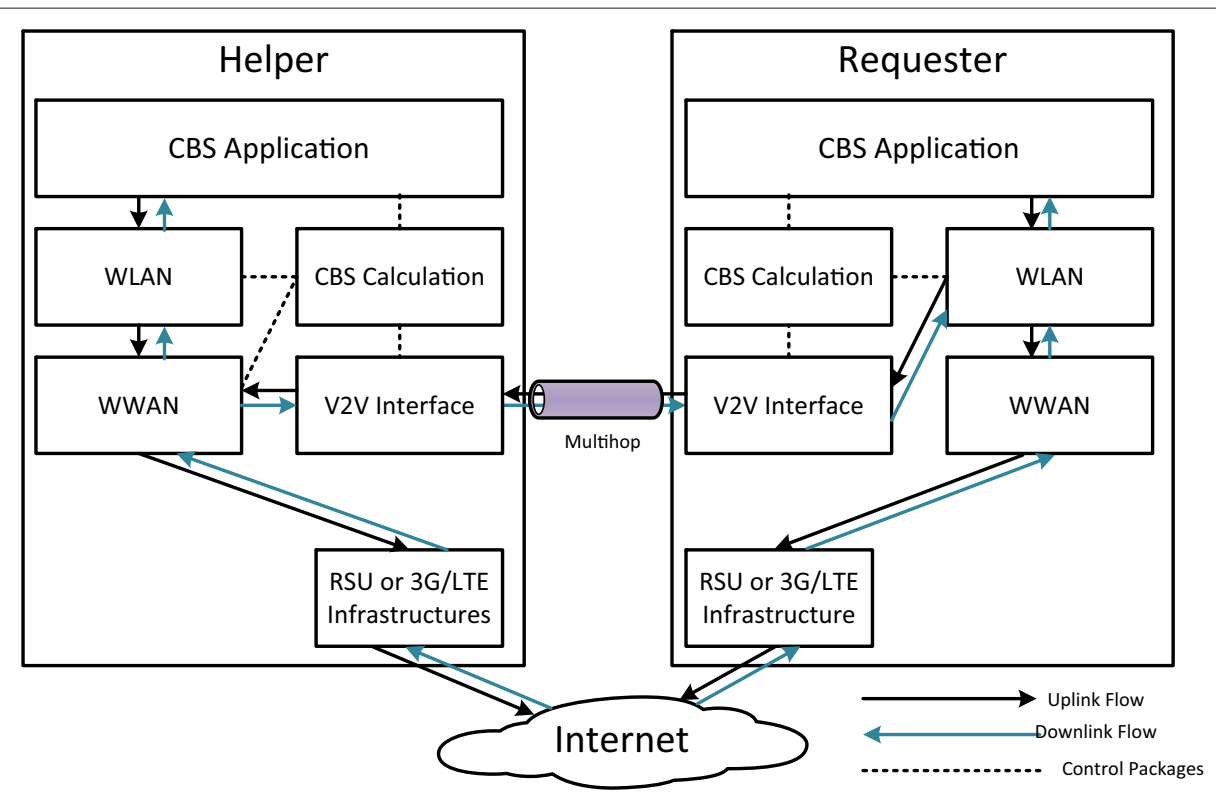

Figure 2 The system architecture for a CBS scenario.

Equations 1 and 2. Equation 1 stands for the objective that every requester prefers to maximize its bandwidth benefit from the helpers' excessive bandwidth, where $U(x)$ is the utility function that usually uses concave monotonically increasing functions to ensure fairness [32].

\section{Table 1 Terms and symbols}

\begin{tabular}{|c|c|}
\hline Symbols & Terms \\
\hline $\mathbf{x}_{i}$ & $\left\{x_{i j}\right\}$ Rate allocation vector of node $i$ \\
\hline$e_{i}$ & $\begin{array}{l}\text { Available link bandwidth between node } i \text { and base station } \\
\text { (BS) tower }\end{array}$ \\
\hline w & Available capacity of WLAN \\
\hline$x_{i i}$ & Flow rate of node $i$ directly connected to BS tower \\
\hline$x_{i j}$ & Flow rate of node $i$ via the link of node $j$ to the BS tower \\
\hline $\mathcal{N}$ & The set of the nodes in CS \\
\hline$x_{i, \max }$ & Max requirement of the traffic flow of node $i$ \\
\hline r & A requester \\
\hline$h$ & A helper \\
\hline$f$ & A forwarder \\
\hline $\mathcal{H}$ & The set of the helpers in CS \\
\hline $\mathcal{R}$ & The set of the requesters in CS \\
\hline$\theta$ & $\begin{array}{l}\text { A path } \theta \triangleq\left\langle f_{0}, f_{1}, \ldots, f_{m}\right\rangle \text {, which is defined as a sequence } \\
\text { of forwarders, each of which, e.g., } f_{i} \text {, receives packets from } \\
\text { its uplink node } f_{i-1} \text { and sends to its downlink node } f_{i+1}\end{array}$ \\
\hline $\mathcal{P}(h, r)$ & The set of all possible paths between $h$ and $r$ \\
\hline$c_{h}, c_{r}$ & $\begin{array}{l}\text { Constant coefficients for helper cost and relay cost per unit } \\
\text { data, respectively }\end{array}$ \\
\hline $\mathcal{L}(\theta)$ & The set of links that compose the path $\theta$ \\
\hline$\delta_{l}$ & $\begin{array}{l}\text { The link cost } \delta_{l} \triangleq \delta(i, j) \triangleq 1 / p(i, j) \text {, which is defined as } \\
\text { the reciprocal of the successful transmission probability } \\
\text { between } i \text { and } j\end{array}$ \\
\hline
\end{tabular}

We use an exponential function as the utility function, i.e., $U(x)=-a^{x}, a \in(0,1)$, for its upward concavity and monotonically increasing feature in the whole real set. Equation 2 expresses the other goal that the V2V communication cost should be minimized. $C_{h r}(x)$ denotes the cost function of a traffic flow $x$ from helper $h$ to requester $r$. The cost function may differ for different kinds of relay schemes, which we will discuss in Section 3.5.

$$
\begin{aligned}
& \max \sum_{i \in \mathcal{H}, j \in \mathcal{R}} U_{1}\left(x_{i j}\right) \\
& \min \sum_{i \in \mathcal{H}, j \in \mathcal{R}} U_{2}\left(C_{i j}\left(x_{i j}\right)\right)
\end{aligned}
$$

Therefore, the problem finally is expressed as that depicted in Table 2, where $\omega_{1}$ and $\omega_{2},\left(\omega_{1}+\omega_{2}=1\right)$, are

Table 2 Problem formulation of the optimal bandwidth sharing among mobile nodes

$$
\max _{\mathbf{x} \geq 0} U(\mathbf{x})=\sum_{i \in \mathcal{H}, j \in \mathcal{R}} U\left(w_{1} x_{i j}-w_{2} C_{i j}\left(x_{i j}\right)\right)
$$

subject to:

$$
\begin{aligned}
0 \leq \sum_{i \in \mathcal{H}} x_{i j} \leq x_{j, \max }, \quad \forall j \in \mathcal{R} \\
\sum_{j \in \mathcal{R}} x_{i j} \leq c_{i}, \quad \forall i \in \mathcal{H} \\
\sum_{i: j \in \mathcal{P}(l)} x_{i j} \leq w, \quad \forall / \in \mathcal{L} \\
x_{i j} \geq 0, \quad \forall(i, j) \in \mathcal{H} \times \mathcal{R}
\end{aligned}
$$


weights to tune the importance relation between objective 1 and objective 2 . They can be referred as the cost weights in the practical system. Thus, the weighted sum expressed in Equation 3 represents the bandwidth utilization subtracting the cost factor in the whole system, which we name as CBS benefit. Equations 4 and 5 depict the two constraints for the optimization, which respectively stand for the following: (1) The total gained bandwidth of each requester shall not exceed its high layer requirement. (2) The total traffic rate via one certain helper's WWAN connection shall not exceed the excessive bandwidth of this link.

It is worthy to mention that there is another constraint that the total $\mathrm{V} 2 \mathrm{~V}$ traffic of the links in an interface range shall not exceed the capacity of the V2V communication channel, which is expressed as Equation 6. However, we assume that the capacity of the $\mathrm{V} 2 \mathrm{~V}$ communication channel is much larger than the WWAN link capacity to simplify the calculation, which means this constraint is not necessary to be considered when solving the optimization problem in Table 2. After solving the problem in Table 2, we verify whether the solution satisfies the constraint of Equation 6 or not. Thus, we can prove the rationality of the assumption.

\subsection{Networking model}

\subsubsection{Topology}

We consider a vehicular ad hoc network in which nodes (vehicles) are spaced along a line. Therefore, the networking model of CBS is simplified as a one-dimensional multihop network from the requester to the helper, which is illustrated in Figure 3a. A requester node 0 borrows bandwidth from the helper node 4 . The traffic flow is forwarded by some or all the forwarders between them, i.e., node 1 to node 3 . There are three relay schemes that can be employed in the scenario, which we will discuss in Section 3.4.

Generally speaking, a requester can borrow bandwidth from a helper for both downloading and uploading. Accordingly, the source and destination of the traffic flow among vehicles are the helper and the requester in the downloading case and the requester and the helper in the uploading case. As the uplink and downlink bandwidth in WWAN are usually considered to be independent, the analysis for the upload case and download case will be same. Therefore, we only focus on the downloading scenario in the following discussion.

To ease presentation, we number the nodes in the order of their sequence along the road, i.e., node $i$ follows node $i+1$ and is followed by node $i-1$. Noting that the index for a node does not mean the network address nor an unchangeable identity for the node, it only represents the node's relative order to the other nodes in a certain period. So when overtaking occurs between two vehicles, the indexes of the two nodes will accordingly change into the new ones.

\subsubsection{Link model}

The link model in the vehicular network may reflect the relationship between the probability of message reception (PMR) and distance. In a simplified model [33], when a node transmits a message, all nodes within distance $r_{T}$ (transmit range) from the sender have the same probability $p(0<p<1)$ of correctly receiving the message in absence of interference. The simplified model can be used to lower bound message reception probability. However, it misses some details of the relationship between PMR to distance, which may be important in our analysis. Thus, we prefer a more accurate model, i.e., the Nakagami radio propagation model, where the actual message reception

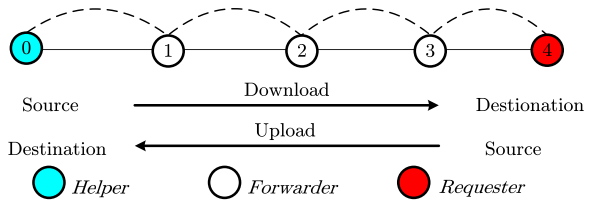

(a)

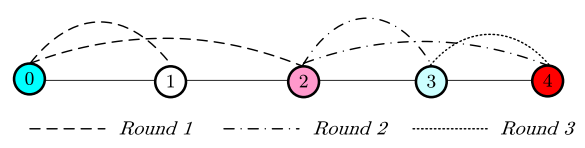

(c)

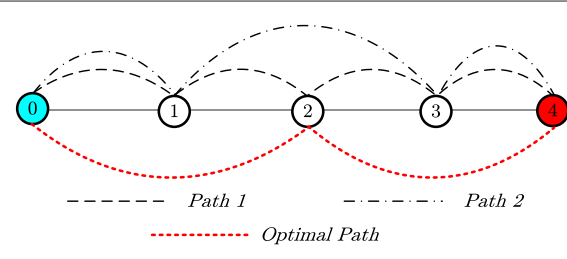

(b)

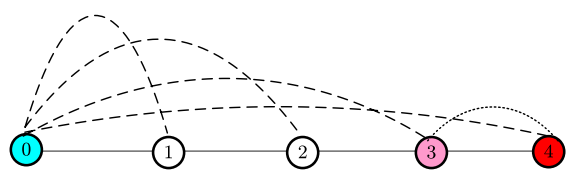

(d)

Figure 3 Networking model and three relay schemes in the CBS scenario. (a) One-dimensional multi-hop networking model. (b) Single relay. (c) Subset relay. (d) All relay. 
probabilities are computed assuming a nominal communication range of $1,000 \mathrm{~m}$ and expressed in Equation 8 .

$$
p(i, j)=P\left(\mu, \frac{\mu}{\omega} C d_{i, j}^{-2 \alpha}\right)
$$

where $P\left(\mu, \frac{\mu}{\omega} x^{2}\right)=\frac{\gamma\left(\mu, \frac{\mu}{\omega} x^{2}\right)}{\Gamma(\mu)}$ is the incomplete gamma function; $p(i, j)$ denotes the PMR of node $i$ and $j, \mu, \omega, C$ and $\alpha$ are constants; and $d_{i, j}$ represents the distance from vehicle $i$ to vehicle $j$. Figure 4 illustrates the relationship between the PMR and distance in the Nakagami model and the simplified model.

\subsubsection{Network traffic}

Since a node with adequate bandwidth will not borrow bandwidth from others, nor a node lacking in bandwidth can lend its own, it is easy to understand that the cooperative bandwidth sharing will not occur if the end-to-end traffic patterns of all mobile nodes are same. Therefore, the network traffic in the cooperative bandwidth sharing scenario is supposed to be unbalanced. We define three kinds of traffic patterns: (i) Idle, which means no network traffic is generated by local applications or services; (ii) Max CBR, which means the local application, e.g., streaming application, expects as much as possible bandwidth that is no more than a maximum constant bit rate (CBR); (iii) Greedy, which means the local application, e.g., file downloading, leeches as much as possible bandwidth and thus generates a bursty traffic. Each type of traffic has a certain portion of nodes to generate. We denote the portions of the nodes that generate the three kinds of traffic as $r_{I}, r_{M}$, and $r_{G}$ respectively, and assume $r_{I}+r_{M}+r_{G}=1$. For $M a x C B R$, the maximum desired bit rate can be either larger or smaller than the nodes' WWAN bandwidth. We further assume that the desired maximum bit rates of the

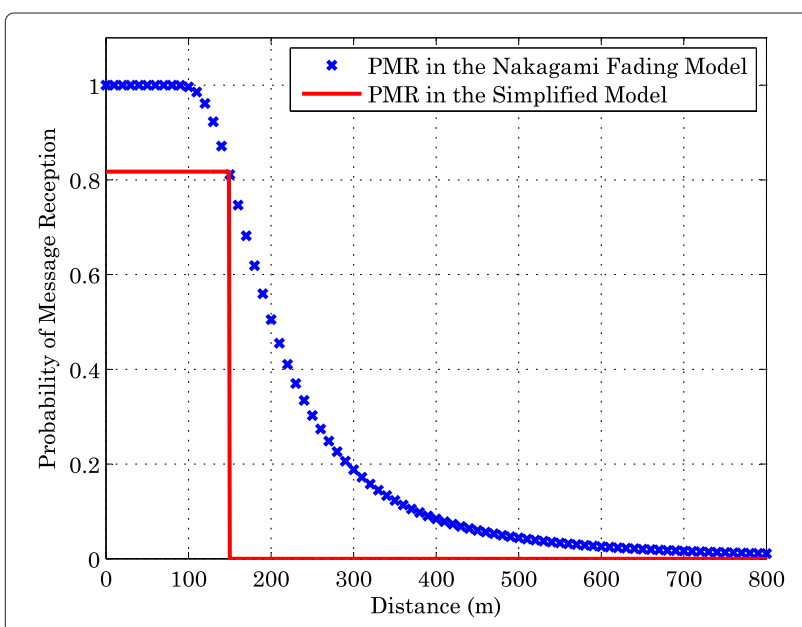

Figure 4 Probability of message reception in the Nakagami model and the simplified model in [33]. nodes that generate $\operatorname{Max} C B R$ traffic obey normal distribution with mean value of $\mu_{e}$ and standard deviation of $\sigma_{e}$.

\subsection{The relay schemes}

Three relay schemes, namely single relay, subset relay, and all relay, are considered in the CBS scenario, which is depicted in Figure 3b,c,d.

\subsubsection{Single relay}

In the single relay scheme, a fixed path $\theta$ between the requester and the helper will be assigned for relaying the traffic, as illustrated in Figure 3b. There will be multiple optional paths between the requester and helper, among which we define an optimal path $\theta_{o}$ as the one with minimum link cost, as interpreted in Equation 9.

Accordingly, we name the single relay scheme using an optimal path $\theta_{o}$ as the optimal single relay scheme. Note that the optimal path may not be unique. However, we consider that all the optimal paths are equivalent as they will produce the same cost value for a certain traffic between the requester and the helper, which we will discuss in Section 3.5.

$$
\forall \theta \neq \theta_{o} \in \mathcal{P}(h, r), \sum_{l \in \mathcal{L}(\theta)} \delta_{l} \geq \sum_{l \in \mathcal{L}\left(\theta_{o}\right)} \delta_{l}
$$

\subsubsection{Subset relay}

Both subset relay and all relay are a kind of opportunistic relay scheme, which takes advantage of the wireless broadcast features. In the subset relay scheme, a node $n$, which may be the source or a forwarder, broadcasts a packet to a subset of forwarders. Among all forwarders that hear the packet, the one which is closest to the destination will further relay the packet to the destination.

A concrete example is depicted in Figure 3c, in which node 0 broadcasts a packet to nodes 1 and 2 and both receive it. During the acknowledgment process, nodes 1 and 2 will agree on node 2 which is closer to the destination. Then, node 2 further forwards the packet to nodes 3 and 4 . However, only node 3 receives the packet. So node 3 finally sends the packet to the destination node 4 . While sending a data packet, a node shall fill the addresses of all the receivers in the subset in order of their distances to the destination and schedule time slots for their acknowledgements in the reverse order. While sending an acknowledgement, a node shall forward its heard acknowledgement whose sender is closest to the destination. In case that no acknowledgement is overheard by a node before its scheduled time to send the acknowledgement, it sends an acknowledgement with its own address and considers itself as the best next forwarder. An illustration of the packet format and transmission process for the example in Figure 3c is shown Figure 5. 


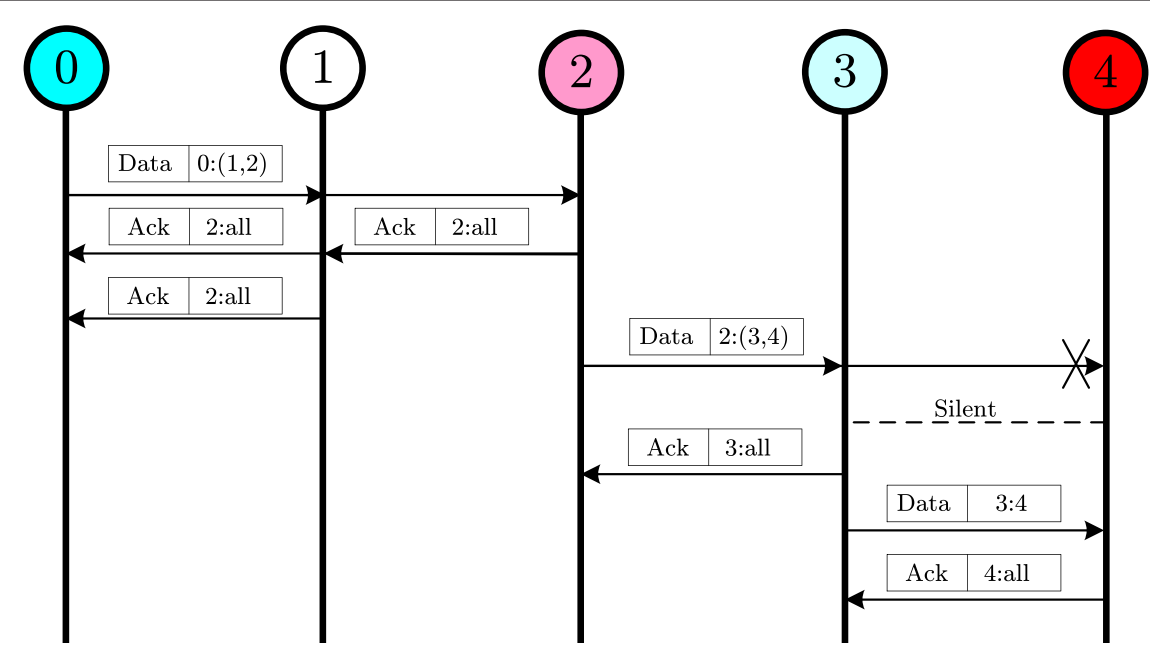

(a)

\begin{tabular}{|c|}
\hline Head \\
\hline Source \\
\hline Destination \\
\hline Address of 1st Node in the Subset \\
\hline Address of 2nd Node in the Subset \\
\hline ... \\
\hline Address of nth Node in the Subset \\
\hline Data \\
\hline CRC Check
\end{tabular}

(b)

\begin{tabular}{|c|}
\hline Head \\
\hline Source \\
\hline Destination \\
\hline Address of ACK Node \\
\hline CRC Check \\
\hline
\end{tabular}

(c)

Figure 5 Packet format and transmission process in the subset relay scheme. (a) Packet transmission process of the example in Figure $3 c$. (b) Data packet illustration for subset relay scheme. (c) Ack packet illustration for subset relay scheme.

Differing from the single relay scheme that uses only one fixed path, the aforementioned subset relay scheme enables the source to use all paths in a subset $\mathcal{P}_{k}(h, r)$ of $\mathcal{P}(h, r) . \mathcal{P}_{k}(h, r)$ can be defined as Equation 10:

$$
\mathcal{P}_{k}(h, r) \triangleq\left\{\theta \mid \theta \in \mathcal{P}(h, r), \forall i \in[0, m), f_{i+1}-f_{i} \leq k\right\}
$$

where $f_{0}=h$ and $f_{m}=r$ and $k$ represents the number of forwarders that are involved in forwarding a packet. Accordingly, we name such a subset relay scheme with value $k$ as a $k$-subset relay scheme. For instance, the $k$ value in the example depicted in Figure 3c is 2.

Therefore, the subset relay scheme opportunistically uses all paths in $\mathcal{P}_{k}(h, r)$, and a path $\theta$ is used only in case that any forwarder $f_{i}$ in the path $\theta$ is the farthest node from the previous forwarder $f_{i-1}$ which successfully receives the packet in range of the $k$ nearest nodes, i.e., $\theta \in \mathcal{P}_{k}(h, r)$ is used iff $\forall f_{i} \in \theta, f_{x} \in\left(f_{i}, f_{i-1}+k\right], f_{i}$ successfully receives from $f_{i-1}$ and $f_{x}$ does not.

\subsubsection{All relay}

The all relay scheme is a special case of the subset relay scheme where the subset of forwarders contains all the forwarders between the requester and the helper, i.e., the $m$-subset relay scheme ${ }^{\mathrm{a}}$. The all relay scheme works almost the same as the subset relay except that there are more participants in forwarding, which is illustrated in Figure 3d.

As a result, the all relay scheme opportunistically uses all possible paths in $\mathcal{P}(h, r)$ in forwarding the traffic. Since in the all relay scheme a packet can be transmitted as near 
to the destination as possible during each transmission round, the scheme can result in the minimum transmission counts, which infers the minimum transmission cost. However, as too many nodes are involved during each transmission round in the all relay scheme, other costs such as computation or local maintenance of neighboring nodes may be high. Noting that the $k$-subset relay scheme limits the maximum transmission range in node counts for each transmission attempts, it may have lower or higher transmission cost than the optimal single relay scheme, which depends on the choice of $k$ and the link qualities of node pairs. Therefore, a $k$-subset relay scheme with a proper $k$ seems to be a trade-off between transmission cost and other overhead, and the transmission cost in the all relay scheme can be used as a lower bound to measure the transmission cost of a certain relay scheme in analysis.

\subsection{Cost model}

It is important that the cost of sharing bandwidth is modeled appropriately, so that the aforementioned optimal solution can suitably reflect balance between requesters' benefit and the expense of the helpers and the possible forwarders.

There are two principal costs that the helpers and the possible forwarders incur in helping the requester. The first cost, the so-called helper cost, is the cost of the helpers' transferring data on the WWAN link for which the WWAN service provider extracts a fee. This fee depends on the tariff structure imposed by the service provider and may depend, in general, on factors such as the user's service plan and the time of day. For our purposes in this paper, we assume that the WWAN tariff is known and is a uniform rate per unit data.

The second cost, namely relay cost, is the opportunity cost of expending computation or power consumption on behalf of a helper and forwarders along the path between the helper and the requester. Although energy and CPU usage are usually considered adequate for mobile devices on vehicles, it is still necessary to define a unified cost per unit data transmission to compensate the contribution of helpers and forwarders for their relaying the traffic. Since retransmissions may occur due to transmission failures, the relay cost on a link of the path from the helper to the requester will be related with the link quality of the corresponding link. Therefore, the overall cost of a traffic flow $x$ between a requester and a helper will be expressed as Equation 11.

$$
C(x)=c_{h} x+c_{r} x \overline{\delta_{\mathcal{P}}}
$$

where $\overline{\delta_{\mathcal{P}}}$ denotes the mean number of transmissions of the packet of the flow $x$ along a path $\theta$ (in the single relay scheme, $\mathcal{P}=\{\theta\}$ ), or a path set $\mathcal{P}$ (in the opportunistic relay schemes).
In the single relay scheme where path $\theta$ is used, the mean transmission number $\overline{\delta_{\theta}}$ can be expressed as $\overline{\delta_{\theta}}=$ $\sum_{l \in \mathcal{L}(\theta)} \delta_{l}$, while in the subset relay scheme or the all relay scheme, the mean transmission number needs to consider all possible paths and the probabilities to use corresponding paths, which is finally expressed in Equation 12:

$$
\begin{aligned}
\overline{\delta_{\mathcal{P}_{k}}} & =\sum_{\theta \in \mathcal{P}_{k}} p_{\theta} \sum_{f_{i} \in \theta} \sum_{j=1}^{\infty} j p\left(f_{i+1}, f_{i}\right) \bar{p}_{f_{i+1}} \bar{p}_{k}^{(j-1)} \\
\bar{p}_{f_{i+1}} & =\prod_{n=f_{i+1}+1}^{f_{i}+k}\left(1-p\left(n, f_{i}\right)\right) \\
\bar{p}_{k} & =\prod_{n=f_{i}+1}^{f_{i}+k}\left(1-p\left(n, f_{i}\right)\right)
\end{aligned}
$$

where $p_{\theta}$ is the probability that path $\theta$ is used, $p\left(f_{i+1}, f_{i}\right)$ denotes the probability that $f_{i+1}$ successfully receives from $f_{i}$ in the $j$ th retransmission, $\bar{p}_{f_{i+1}}$ denotes the probability that no farther node than $f_{i+1}$ receives from $f_{i}$ in the $j$ th retransmission, and $\bar{p}_{k}^{(j-1)}$ represents that none of the $k$ nearest nodes from $f_{i}$ receives from $f_{i}$ for first $j-1$ transmission attempts.

Therefore, with known topology and the successful transmission probabilities of any two node pairs, the cost of a flow from the helper to the requester in different relay schemes can be figured out.

\subsection{Vehicular flow model}

In order to investigate the OCS, vehicular traffic needs to be modeled so that the intervals between vehicles and the link property can be estimated. We consider a microscopic model of the road traffic. More specifically, we consider a car-following model [34] with $N$ vehicles for a singleline traffic flow on a circular road, where very vehicle in the system follows one vehicle and is followed by another vehicle in the system.

Consider the system depicted in Figure 6, which models $N$ vehicles on a circular road of the length $L$. The pairs $\left(y_{i}, z_{i}\right)$ denote the position $y_{i}$ along the road and the velocity $z_{i}$ of the vehicle number $i$ in the system. The acceleration $\dot{z}_{i}$ of each vehicle depends on the difference between the vehicle's current velocity $z_{i}$ and the optimal velocity function $V=V\left(y_{i+1}-y_{i}\right)$. Therefore, the dynamics of traffic flows is expressed in Equation 15.

$$
\begin{gathered}
\dot{y}_{i}=z_{i}, \quad \dot{z}_{i}=\frac{1}{\tau}\left[V\left(y_{i+1}-y_{i}\right)-z_{i}\right], \\
y_{N+1}=y_{1}+L, \quad i=1, \ldots, N .
\end{gathered}
$$

The optimal velocity function $d \mapsto V(d)$, with domain $d \geq 0$, satisfies the following assumptions: $V$ is positively valued and monotonically increasing, $V(0)=0$, $\lim _{d \rightarrow \infty} V(d)=V^{\max }>0$, and there exists a positive 


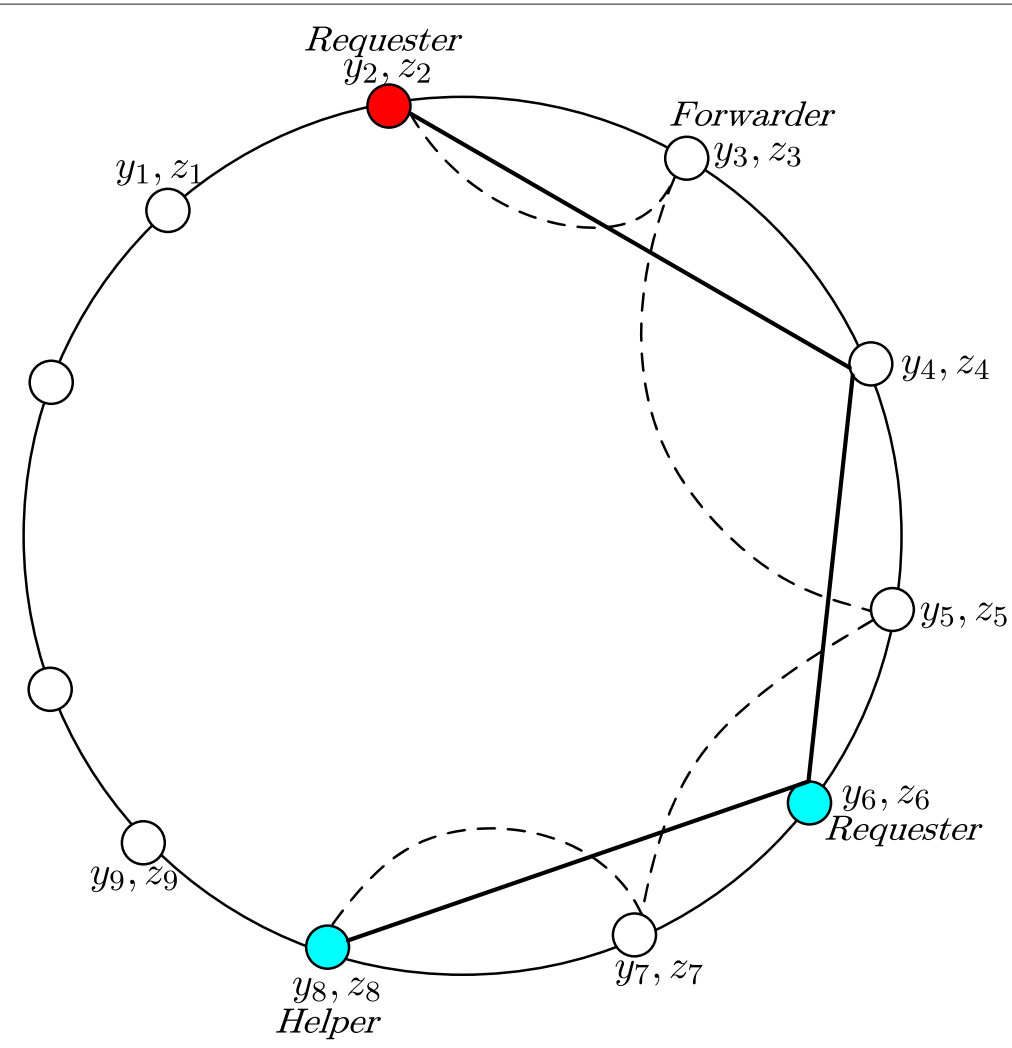

Figure 6 The car-following model for a single-line traffic flow on a circular road.

constant $b$ such that $\ddot{V}(d)>0,(\ddot{V}(d)<0)$ if $d<b,(d>$ $b)$ (see $[35,36])$. The reciprocal value of the parameter $\tau$ is called sensitivity [37]. In all forthcoming computations, we will consider the hyperbolic optimal velocity function defined as

$$
V(d)=V^{\max } \frac{\tanh (a(d-1))+\tanh (a)}{1+\tanh (a)},
$$

where $V^{\max }$ and $a$ are positive constants [37]. The choice of $V$ imposes a driving law, and we assume that this law is the same for all $N$ drivers. The difference $d_{i} \triangleq y_{i+1}-y_{i}, i=$ $1, \ldots, N$ is called headway of the $i$ th vehicle, which will be also used in the link quality calculation as depicted in Equation 8.

With the aforementioned definitions, given an initial condition $\left[\mathbf{y}^{0}, \mathbf{z}^{0}\right] \in \mathbb{R}^{N} \times \mathbb{R}^{N}$, the system in Equation 15 defines a flow on $\mathbb{R}^{N} \times \mathbb{R}^{N}$. Without loss of generality, we assume

$$
\begin{aligned}
& s \leq y_{1}^{0} \leq y_{2}^{0} \leq \cdots \leq y_{N-1}^{0} \leq y_{N}^{0} \leq L+s, \\
& \mathbf{z}^{0}=\left(z_{1}^{0}, \ldots, z_{N}^{0}\right), \quad z_{i}^{0}>0, i=1, \ldots, N,
\end{aligned}
$$

where $s \in \mathbb{R}$ is an arbitrary phase shift.

Therefore, given an initial condition $\left[\mathbf{y}^{0}, \mathbf{z}^{0}\right]$ and the network traffic patterns for vehicular nodes, the parameters of the problem formulation depicted in Table 2 can be determined by using numerical calculation. We can then solve the optimal bandwidth sharing problem and find the features of the number of hops in the optimal solution. Thus, we can recommend the OCS for cooperative bandwidth sharing.

\section{Distributed algorithm}

For practical consideration, the problem depicted in Table 2 needs to be solved in a distributed way to achieve optimal cooperative bandwidth sharing. Thus, we briefly introduce the distributed algorithm in this section.

We use the dual decomposition method introduced in [32] as follows. Clearly, if the constraint Equations 4 to 7 were absent, then the problem would decouple. Therefore, it makes sense to form the Lagrangian by relaxing the coupling constraint in Table 2 as follows:

$$
\begin{aligned}
L(\mathbf{x}, \lambda)= & \sum_{(i, j) \in \mathcal{H} \times \mathcal{R}} U\left(w_{1} x_{i j}-w_{2} C_{i j}\left(x_{i j}\right)\right) \\
& +\sum_{j \in \mathcal{R}}\left(\alpha_{j}\left(x_{j, \max }-\sum_{i \in \mathcal{H}} x_{i j}\right)\right) \\
& +\sum_{i \in \mathcal{H}}\left(\beta_{i}\left(c_{i}-\sum_{j \in \mathcal{R}} x_{i j}\right)\right) \\
= & \sum_{\mathcal{H} \times \mathcal{R}} L_{i j}\left(x_{i j}, \lambda_{i j}\right)+\sum_{\mathcal{H}} \beta_{i} c_{i}+\sum_{\mathcal{R}} \alpha_{j} x_{j, \max }
\end{aligned}
$$


where $\lambda$, which includes $\alpha, \beta$, is the Lagrange multiplier vector, and $L_{i j}\left(x_{i j}, \lambda_{i j}\right)=-a^{\omega_{i j} x_{i j}}-\left(\alpha_{j}+\beta_{i}\right) x_{i j}$ is the Lagrangian to be maximized by the local source node $i$ for each source-destination pair $i \rightarrow j$.

The dual decomposition results in each node $i$ solving, for the given $\lambda_{(i j)}$

$$
x_{i j}^{*}\left(\lambda_{i j}\right)=\arg \max _{x_{i j} \geq 0}\left[L_{i}\left(x_{i j}, \lambda_{i j}\right)\right], \forall(i, j) \in \mathcal{H} \times \mathcal{R}
$$

which is unique due to the strict concavity of the object function.

The master dual problem is

$$
\min _{\lambda} g(\lambda)=\sum_{i j \in \mathcal{H} \times \mathcal{R}} g_{i j}\left(\lambda_{i j}\right)+\lambda^{T} \mathbf{c}
$$

subject to $\lambda \geq 0$

where $g_{i j}\left(\lambda_{i j}\right)=L_{i j}\left(x_{i j}^{*}\left(\lambda_{i j}\right), \lambda_{i j}\right)$ and $\mathbf{c}$ is a constant vector where $\lambda^{T} \mathbf{c}=\sum_{\mathcal{H}} \beta_{i} c_{i}+\sum_{\mathcal{R}} \alpha_{j} x_{j \text {,max }}$. Since the solution in Equation 20 is unique, the following subgradient method can be used:

$$
\begin{aligned}
& \alpha_{j}(t+1)=\left[\alpha_{j}(t)-\delta_{1}(t)\left(x_{j, \max }-\sum_{i \in \mathcal{H}} x_{i j}\right)\right]^{+} \\
& \beta_{i}(t+1)=\left[\beta_{i}(t)-\delta_{2}(t)\left(c_{i}-\sum_{j \in \mathcal{R}} x_{i j}\right)\right]^{+}
\end{aligned}
$$

where $t$ is the iteration index, $\delta(t)$ is a sufficiently small positive step size, and $[\cdot]^{+}$denotes the projection onto the nonnegative orthant.

In summary, we have the following algorithm.

\section{Algorithm 1 The distributed dual algorithm for solving} the problem depicted in Table 2

1: Set $t=0$ and $\lambda_{i j}$ equals some nonnegative value for all nodes;

2: Each source locally solves its problem by computing Equation 20 and then broadcasts the solution $x_{i j}^{*}\left(\lambda_{i j}(t)\right)$;

3: Each link updates its prices with the gradient iterate Equations 22 and 23 and broadcasts the new price $\lambda_{i j}(t+1)$

4: Set $t \leftarrow t+1$ and go to step 2;

\section{Results analysis}

Intuitively, whether it is worthy to use multi-hop bandwidth sharing or not, the optimal hop range may be different with parameters and scenarios. Therefore, we investigate the results based on the proposed model depicted in Section 3 with different settings. Combina- tions of different network traffic patterns, relay schemes, cost weights, and vehicular flow settings are input to the model, and the distribution number of hops and the cooperation scope as the outputs are particularly concerned in the analysis. The input settings for the model are listed in Table 3, where the default value marked with a star of each setting is used when another parameter is being tuned and studied.

\subsection{Network traffic patterns}

In Section 3, we simplified the network traffic types of nodes as Idle, Max CBR, and Greedy. We set the mean $\mu_{e}$ and standard deviation $\sigma_{e}$ of the traffic demand of Max CBR nodes to be equal to the WWAN capacity and tune the portion of the idle nodes in the CBS scenario. The relation of the OCS between the requester and the helper over the portion $r_{I}$ is illustrated in Figure $7 \mathrm{a}^{\mathrm{b}}$. Generally speaking, while the portion of the Idle nodes $r_{I}$ increases, the number of possible helpers increases. However, it is interesting to find that the maximum number of hops between the requester and the helper in the optimal solution does not change. Thus, we believe that the number of possible helpers is not one of the main factors that determine whether a requester can get help or not.

We also fixed $r_{I}$ and tune the portions $r_{M}$ and $r_{G}$. See Figure $7 b, c$. We have the same conclusion that the background traffic is not important to the cooperative scope, except the extreme case that most nodes are greedy, which can be excluded from considerations in designing the CBS system. Therefore, these results hint that we can exclude the background traffic from the considerations of deciding the CS when designing a multihop CBS scheme, which also means that a fixed CS can be applied despite of the dynamics of background traffic.

\subsection{Relay schemes}

The choice of relay schemes impacts the optimal cooperation scope because it results in different levels of transmission cost. To better understand the effect of relay schemes,

Table 3 Parameters and settings in the numeric analysis

\begin{tabular}{ll}
\hline Parameters & Values \\
\hline Portion of nodes with different & $\begin{array}{l}0.1 \text { to } 0.9 \text {, default } r_{l}=0.5, r_{M}= \\
\text { traffic }\left(r_{1}, r_{M}, r_{G}\right)\end{array}$ \\
WLAN data rate (Mbps) & 54 \\
WWAN link rate (Kbps) & 1,024 \\
Relay schemes & $\begin{array}{l}\text { Optimal single relay, } k \text {-subset } \\
\text { relay (default } k=3) \text {, all relay }\end{array}$ \\
$\begin{array}{l}\text { Vehicular density ( } \rho \text {, vehicles per } \\
\text { kilometer) }\end{array}$ & 5 to 20 , default $\rho=10$ \\
\end{tabular}




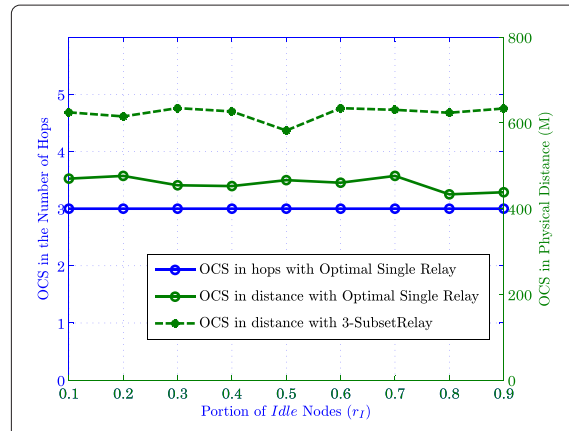

(a)

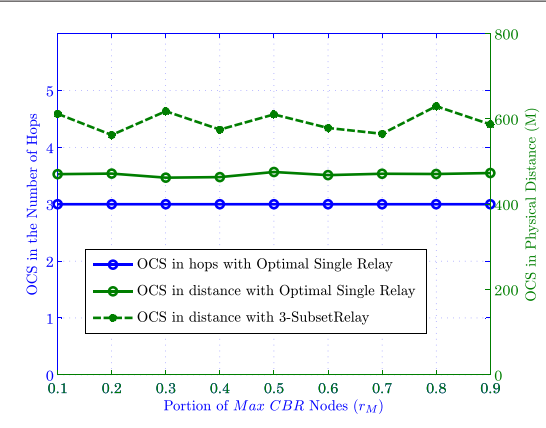

(b)

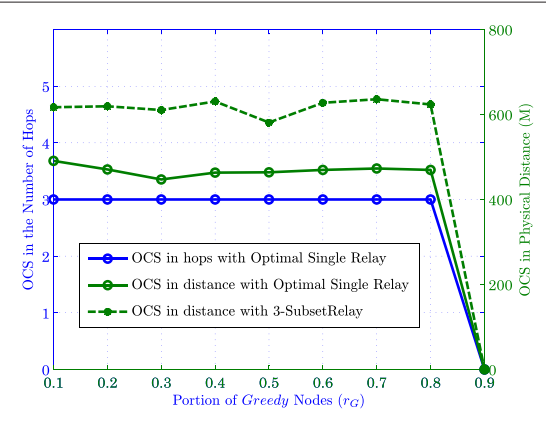

(c)

Figure 7 The OCS over the varied portions of nodes with different network traffic. (a) The OCS over the portion of the /dle nodes $r /$. $\left(r_{M}=r_{G}=\frac{1-r_{l}}{2}\right)$. (b) The OCS over the portion of the Max CBR nodes $r_{M} .\left(r_{l}=r_{G}=\frac{1-r_{M}}{2}\right)$. (c) The OCS over the portion of the Greedy nodes $r_{G}$. $\left(r_{l}=r_{M}=\frac{1-r_{G}}{2}\right)$.

we first briefly look at the transmission cost of a packet transmitted from a node $i$ to a node $i+j$ between which there are $n=j-1$ nodes that can be used as relay nodes. Figure $8 \mathrm{a}, \mathrm{b}$ depicts the transmission cost over the number of nodes between them, $n$. Cases in both high node density scenario and low node density scenario are studied.

As is mentioned in Section 3.4, the all relay scheme reflects the lower bound of the transmission cost, which is proved true in both cases from Figure 8a,b. Meanwhile, for a $k$-subset relay scheme, the transmission cost will decrease and approach the results of the all relay as $k$ increases.

When comparing the $k$-subset relay scheme with the optimal single relay scheme, we can find difference in the high node density scenario and the low node density scenario. In the illustrated result of a low node density scenario depicted in Figure $8 \mathrm{~b}$, the transmission cost in a $k$-subset relay scheme $(k>1)$ is lower than that in the optimal single relay scheme. While in the high node density scenario result depicted in Figure 8a, the transmission cost in a $k$-subset relay scheme is lower than only when $k$ is large enough. It is because the $k$-subset relay scheme may limit the maximum transmission range for each transmission attempt even if the link quality is good enough to transmit the packet to the destination. Therefore, the results depicted in Figure 8a,b can be explained as the node density affects the link qualities between nodes and the $k$-subset relay scheme's limitation. On the contrary, the $k$-subset relay scheme can show

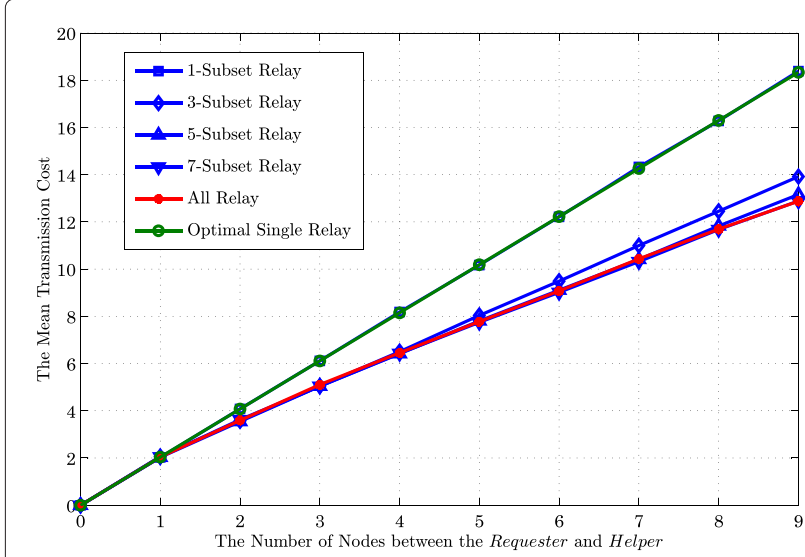

(a)

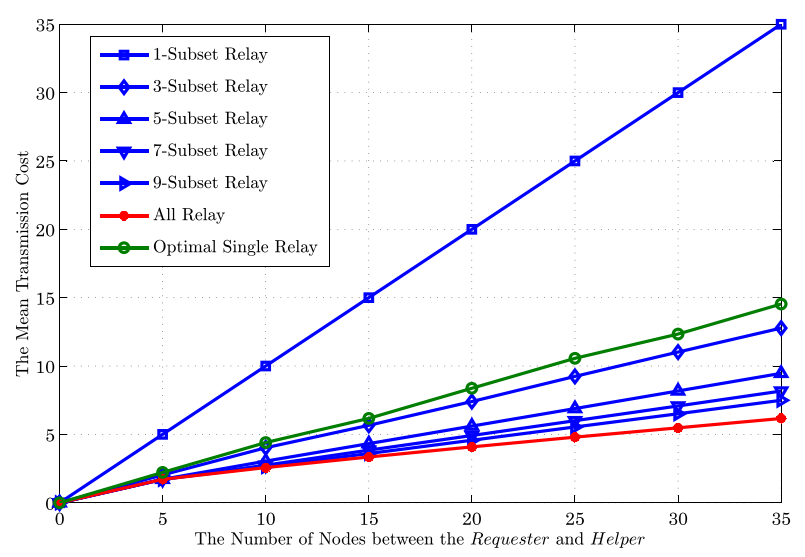

(b)

Figure 8 Packet transmission cost with different relay schemes. (a) Packet transmission cost with different relay schemes in low node density scenario $(\rho=5)$. (b) Packet transmission cost with different relay schemes in high node density scenario $(\rho=20)$. 


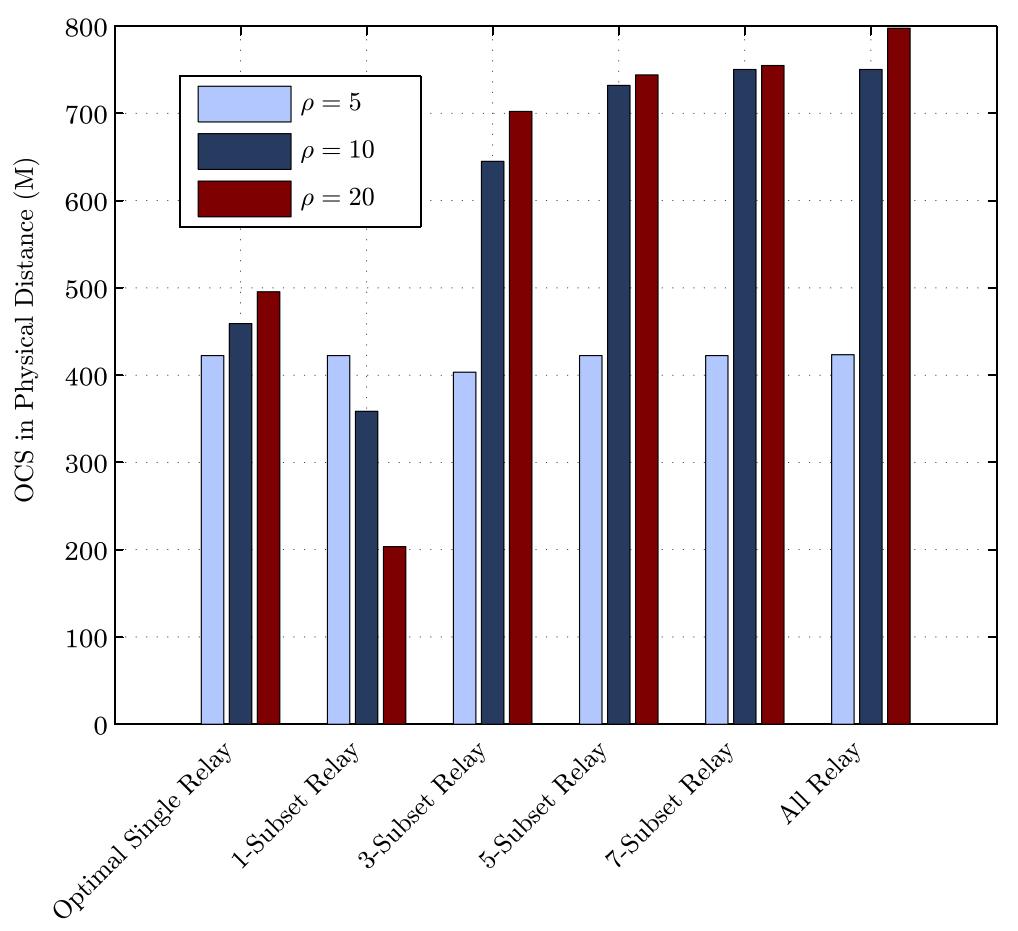

Figure 9 The OCS in physical distance with different relay schemes.

its predominance of multiple opportunistic relay paths over a single relay scheme when the link quality is not promising.

With the knowledge of the impact of the relay schemes on the transmission cost, we can then understand the relationship between the cooperation scope and the relay scheme shown in Figure 9. In a same cooperative bandwidth sharing scenario, the OCS with the all relay scheme will be the largest one. The OCS with the $k$-subset relay scheme is a little bit larger than that with the optimal single relay scheme when node density is low and smaller in the opposite condition.

\subsection{Cost weights}

The cost weights, $\omega_{1}$ and $\omega_{2}$, as depicted in Equation 3, are some subjective factors that reflect the degree of

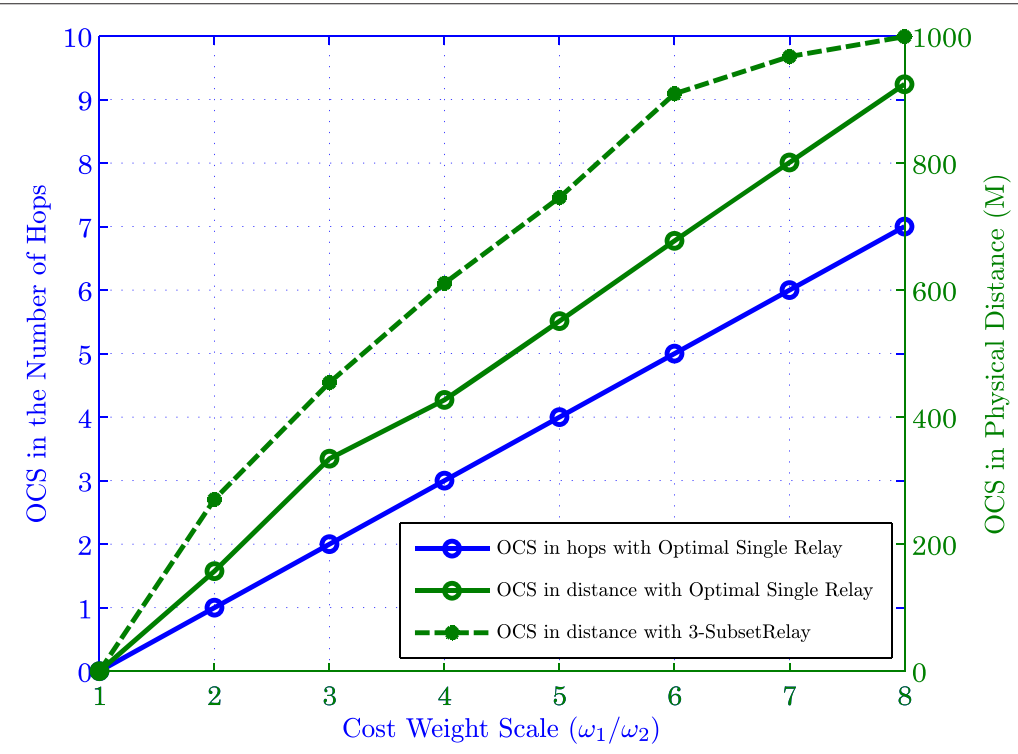

Figure 10 The OCS over different cost weight settings. 


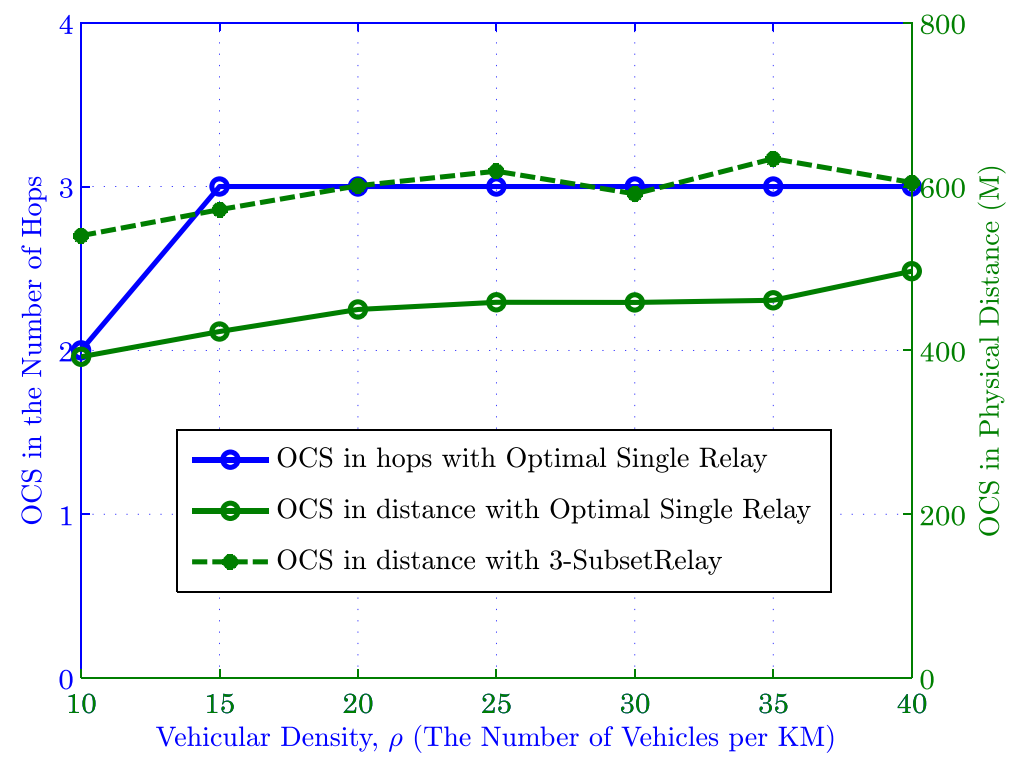

Figure 11 The OCS over different vehicular density.

willingness to encourage cooperative bandwidth sharing. It may be defined with the accounting and pricing policies when the scheme is applied. When a smaller $\omega_{2}$ is applied, the cooperative bandwidth sharing system will tend to utilize more bandwidth of all nodes and may be less sensitive to the multi-hop cost. Similarly, larger $\omega_{2}$ will magnify the overhead of multi-hop cooperation and thus result in a smaller cooperation scope. The results of the cost weight impact is shown in Figure 10.

\subsection{Vehicular flow}

Finally, the impact of vehicular flow feature is investigated. We studied the optimal cooperation scope under vehicular flows with different vehicular densities. Based on the vehicular flow model, different vehicular densities lead to different distances between nodes' pairs as well as the velocities. Consequently, the link quality between nodes and the transmission cost may also vary. Figure 11 compares the OSC over different vehicular densities.

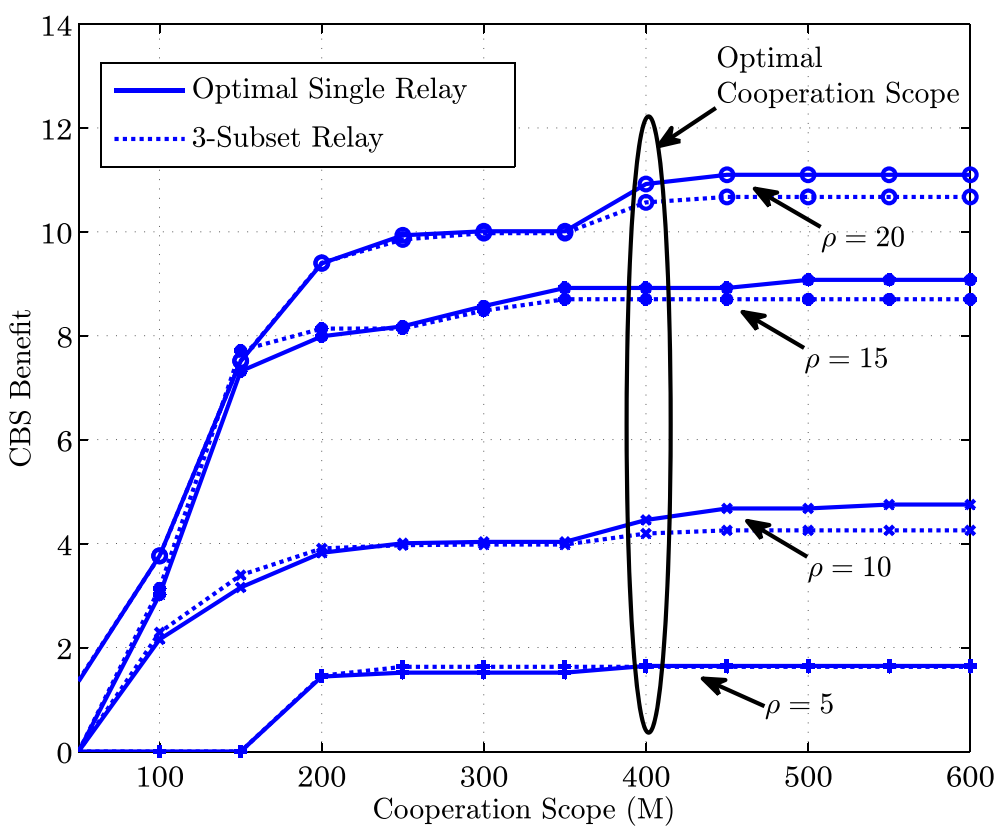

Figure 12 The cooperation benefits over vehicular density. 
It is interesting to find that the optimal cooperation scopes do not change too much with the vehicular density. This can be explained as the cooperation scope may be decided only by propagation feature despite of the number of nodes in this scope. However, when the density goes high, the number of nodes in the same cooperation scope increases. Thus, a requestor may get more benefit from cooperative bandwidth sharing. On the contrary, when the density decreases, a requestor may get less benefit from cooperative bandwidth sharing. But in such low density scenario, increasing the cooperation scope does not help to improve the benefit of CBS. Therefore, the optimal cooperation scope remains the same as that in the high density scenario with relatively less benefit. Figure 12 shows such relations between the cooperation scope and the benefit, in which the optimal cooperation scope is about $400 \mathrm{M}$ despite the vehicular density.

\section{Conclusion}

In this paper, we have proposed an analytical model that tries to reveal the optimal cooperation scope for cooperative bandwidth sharing in vehicular networks. Four components of the model, which are the network model, relay schemes, cost model, and vehicular flow model, are studied. The optimal cooperation scope is solved as the maximum physical distance or hops between the requester and the helper in the optimal solution to maximize the CBS benefit. The results show that the optimal cooperation scope is sensitive to the cost weight and may also vary with different relay schemes. The vehicular density does not affect the optimal cooperation scope much but can result in different CBS benefit. The model and findings of the relations between optimal CS and the running environment revealed in this paper can be used as a reference for parameter choosing when designing the CBS system in vehicular networks.

\section{Endnotes}

${ }^{a}$ Note that the single relay scheme is not a 1-subset relay.

${ }^{\mathrm{b}} \mathrm{OCS}$ in the number of hops with the $k$-subset relay scheme is ignored as the hops may vary in an opportunistic relay scheme.

\section{Competing interests}

The authors declare that they have no competing interests.

\section{Acknowledgements}

The research is supported by the National Science Council of the Republic of China under Grant No. NSC 102-2221-E-006-009 and National Science Fund of China (Grant No. 61202303).

\section{Author details}

${ }^{1}$ Department of EIE, Huazhong University of Science and Technology, Luoyu Rd. 1037, Wuhan 430074, China. ${ }^{2}$ Department of CSIE, National Cheng Kung University, Tainan 701, Taiwan.
Received: 30 January 2014 Accepted: 24 June 2014

Published: 11 August 2014

\section{References}

1. X Wu, S Subramanian, R Guha, RG White, J Li, KW Lu, A Bucceri, T Zhang, Vehicular communications using DSRC: challenges, enhancements, and evolution. Select. Areas Commun. IEEE J. 31(9), 399-408 (2013)

2. JB Kenney, Dedicated short-range communications (DSRC) standards in the United States. Proc. IEEE. 99(7), 1162-1182 (2011)

3. L Tu, C-M Huang, Forwards: a map-free intersection collision-warning system for all road patterns. Vehicular Technol. IEEE Trans. 59(7), 3233-3248 (2010)

4. H-R Tseng, S-K Tseng, T-H Su, P-C Kang, Design and implementation of wave/DSRC payment systems, in 2012 12th International Conference on ITS Telecommunications (ITST). Taipei, Taiwan, 5-8 Nov. 2012 (2012), pp. 66-70

5. D Niyato, P Wang, Optimization of the mobile router and traffic sources in vehicular delay-tolerant network. Vehicular Technol. IEEE Trans. 58(9), 5095-5104 (2009)

6. S Pack, T Kwon, Y Choi, EK Paik, An adaptive network mobility support protocol in Hierarchical Mobile IPv6 networks. Vehicular Technol. IEEE Trans. 58(7), 3627-3639 (2009)

7. J Yao, SS Kanhere, M Hassan, Improving QoS in high-speed mobility using bandwidth maps. Mobile Comput. IEEE Trans. 11(4), 603-617 (2012)

8. A Balasubramanian, R Mahajan, A Venkataramani, BN Levine, J Zahorjan, Interactive WiFi connectivity for moving vehicles, in Proceedings of the ACM SIGCOMM 2008 Conference on Data Communication, SIGCOMM '08 (ACM New York, 2008), pp. 427-438

9. SCespedes, X Shen, C Lazo, IP mobility management for vehicular communication networks: challenges and solutions. Commun. Mag. IEEE. 49(5), 187-194 (2011). doi:10.1109/MCOM.2011.5762817

10. V Devarapalli, R Wakikawa, A Petrescu, P Thubert, Network mobility (NEMO) basic support protocol (2005). RFC 3963, Jan. http://tools.ietf.org/ $\mathrm{html} / \mathrm{rfc} 3963$

11. D Johnson, C Perkins, J Arkko, et al., Mobility support in IPv6. RFC 3775 , June (2004). http://www.ietf.org/rfc/rfc3775.txt

12. J Montavont, J Lorchat, T Noel, Deploying NEMO: a practical approach, in 2006 6th International Conference on ITS Telecommunications Proceedings (Chengdu, China. 23 June 2006 (2006), pp. 1053-1056, doi:10.1109/ITST.2006.288768

13. J-H Lee, T Ernst, Lightweight network mobility within PMIPv6 for transportation systems. Syst. J. IEEE. 5(3), 352-361 (2011). doi:10.1109/JSYST.2011.2158681

14. SIslam, AH Abdalla, MH Habaebi, SA Latif, MK Hasan, RA Saeed, Multihoming based mobility management scheme in NEMO: qualitative and quantitative analysis, in 2013 International Conference on Computing, Electrical and Electronics Engineering (ICCEEE) Khartoum, Sudan, 2013). Aug. 2013 (2013), pp. 394-397, doi:10.1109/ICCEEE.2013.6633969

15. K Chebrolu, RR Rao, Bandwidth aggregation for real-time applications in heterogeneous wireless networks. IEEE Trans. Mobile Comput. 5(4), 388-403 (2006)

16. P Rodriguez, R Chakravorty, J Chesterfield, I Pratt, S Banerjee, MAR: a commuter router infrastructure for the mobile internet, in Proceedings of the 2nd International Conference on Mobile Systems, Applications, and Services, Mobisys '04 (ACM New York, 2004), pp. 217-230

17. S Kandula, D Katabi, S Sinha, A Berger, Dynamic load balancing without packet reordering. SIGCOMM Comput. Commun. Rev. 37(2), 51-62 (2007)

18. V Sharma, K Kar, KK Ramakrishnan, S Kalyanaraman, A transport protocol to exploit multipath diversity in wireless networks. Netw. IEEE/ACM Trans. 20(4), 1024-1039 (2012)

19. TB S Kandula, K Ching-Ju Lin, D Katabi, FatVAP: aggregating AP backhaul capacity to maximize throughput, in 5th USENIX Symposium on Networked Systems Design and Implementation (San Francisco, CA, 2008)

20. G Ananthanarayanan, VN Padmanabhan, L Ravindranath, CA Thekkath, COMBINE: leveraging the power of wireless peers through collaborative downloading, in Proceedings of the 5th International Conference on Mobile Systems, Applications and Services, MobiSys '07 (ACM New York, 2007), pp. 286-298

21. L Badia, N Bui, M Miozzo, M Rossi, M Zorzi, On the exploitation of user aggregation strategies in heterogeneous wireless networks, in Proceedings of the 11th International Workshop on Computer-Aided 
Modeling, Analysis and Design of Communication Links and Networks (Trento, Italy. 8-9 June 2006 (2006), pp. 8-15

22. R Baldessari, A Festag, J Abeille, NEMO meets VANET: a deployability analysis of network mobility in vehicular communication, in 7th International Conference on ITS Telecommunications, 2007. ITST'07 Sophia Antipolis, France. 6-8 June 2007 (2007), pp. 1-6, doi:10.1109/ITST.2007.4295897

23. H Soliman, L Bellier, K Elmalki, C Castelluccia, Hierarchical mobile IPv6 (HMIPv6) mobility management (2008). RFC 5380, Oct. http://tools.ietf. org/html/rfc5380

24. VDKC S Gundavelli, K Leung, B Patil, Proxy Mobile IPv6 (2005). RFC 5213, Aug. http://tools.ietf.org/html//rfc5213

25. J-H Lee, T Ernst, N Chilamkurti, Performance analysis of PMIPv6-based network mobility for intelligent transportation systems. Vehicular Technol. IEEE Trans. 61(1), 74-85 (2012). doi:10.1109/TVT.2011.2157949

26. MS Hossain, M Atiquzzaman, W Ivancic, Performance evaluation of multihomed NEMO, in 2012 IEEE International Conference on Communications (ICC) Ottawa, Canada. 10-15 June 2012 (2012), pp. 5429-5433, doi:10.1109//CC.2012.6364465

27. R Johari, N Gupta, S Aneja, CACBR: Context aware community based routing for intermittently connected network, in Proceedings of the 10th ACM Symposium on Performance Evaluation of Wireless Ad Hoc, Sensor, \& Ubiquitous Networks. PE-WASUN '13 ACM New York, 2013), pp. 137-140

28. E Jung, Y Wang, I Prilepov, F Maker, X Liu, V Akella, User-profile-driven collaborative bandwidth sharing on mobile phones, in MCS '10: Proceedings of the 1st ACM Workshop on Mobile Cloud Computing \& Services (ACM New York, 2010), pp. 1-9

29. T Taleb, K Hashimoto, MS2: a new real-time multi-source mobile-streaming architecture. Broadcasting IEEE Trans. 57(3), 662-673 (2011)

30. L McNamara, C Mascolo, L Capra, Media sharing based on colocation prediction in urban transport, in Proceedings of the 14th ACM International Conference on Mobile Computing and Networking, MobiCom '08 (ACM New York, 2008), pp. 58-69

31. L Tu, C-M Huang, Collaborative content fetching using MAC layer multicast in wireless mobile networks. Broadcasting IEEE Trans. 57(3), 695-706 (2011)

32. DP Palomar, M Chiang, A tutorial on decomposition methods for network utility maximization. IEEE J. Select. Areas Commun. 24(8), 1439-1451 (2006)

33. G Resta, P Santi, J Simon, Analysis of multi-hop emergency message propagation in vehicular ad hoc networks, in MobiHoc '07: Proceedings of the 8th ACM International Symposium on Mobile Ad Hoc Networking and Computing (ACM New York, 2007), pp. 140-149

34. RW Rothery, Car following models. Trac Flow Theory (1992). https://www. tfhrc.gov/publications/research/operations/tft/chap4.pdf

35. L Buric, $V$ Janovskỳ, A road traffic model with overtaking: continuation of the oscillatory patterns. Numerical. Math. Adv. Appl, 753-760 (2008). doi:10.1007/978-3-540-69777-0_90

36. M Bando, K Hasebe, A Nakayama, A Shibata, Y Sugiyama, Dynamical model of traffic congestion and numerical simulation. Phys. Rev. E. 51(2), 1035-1042 (1995)

37. M Bando, K Hasebe, K Nakanishi, A Nakayama, A Shibata, Y Sugiyama, Phenomenological study of dynamical model of traffic flow. J de Physique I. 5(11), 1389-1399 (1995)

doi:10.1186/1687-1499-2014-130

Cite this article as: Tu et al.: Analysis of the scope of cooperative bandwidth sharing among mobile routers in vehicular networks. EURASIP Journal on Wireless Communications and Networking 2014 2014:130.

\section{Submit your manuscript to a SpringerOpen ${ }^{\circ}$ journal and benefit from:}

- Convenient online submission

- Rigorous peer review

- Immediate publication on acceptance

- Open access: articles freely available online

- High visibility within the field

- Retaining the copyright to your article

Submit your next manuscript at $\boldsymbol{~ s p r i n g e r o p e n . c o m ~}$ 